\title{
Debatable points in the anatomy of the lower oesophagus
}

\author{
G. W. FRIEDLAN D ${ }^{1}$, D. H. MELCHER, F. R. BERRIDGE, \\ AND G. A. GRESHAM \\ From the Department of Radiodiagnosis, Addenbrooke's Hospital, Cambridge, \\ and the Pathology Department, University of Cambridge
}

\begin{abstract}
Although there are a number of accounts of the anatomy of the distal oesophagus in the literature, none is definitive. The arrangement of the muscle fibres, the structure and nature of the sphincter mechanism, the mode of attachment of the mucosa to the muscle, the cause of the 'mucosal junction' ring, the factors which keep the stomach in the abdomen, and the anatomical criteria of a sliding hiatal hernia are all still sub judice. For this reason, a careful anatomical study was carried out on 132 post-mortem specimens of oesophagus, stomach, and diaphragm. One hundred and twenty-five specimens were adjudged normal. There were seven cases of hiatal hernia, one of which had associated columnar-cell lining of the oesophagus and Barrett's ulcers. A wide spectrum of ages was represented, ranging from new-born to 102 years.

In this paper, certain practical aspects of the anatomy of the distal oesophagus are discussed and a number of new findings are presented. No attempt will be made to review the literature.
\end{abstract}

\section{MUSCLES OF THE OESOPHAGUS}

The muscularis propria of the oesophagus consists of two principal muscle coats, an inner transverse and outer longitudinal layer. Additional muscle bundles which occur in this region are the sling fibres of the stomach, the 'constrictor cardiae', and the inconstant bracket fibres.

THE TRANSVERSE AND LONGITUDINAL MUSCLE LAYERS The transverse muscle layer is really a reticular layer, for each muscle bundle both contributes to and receives from adjacent muscle bundles (Figs 1a and b). At irregular intervals the transverse layer contributes muscle bundles to

1 Present address : The Hospital for Sick Children. Great Ormond Wtreet, London, W.C.1, and University College Hospital, London, the longitudinal layer (Fig. 2). This anatomical continuity may account for the fact that these two layers contract simultaneously in living persons.

THE SLING FIBRES OF THE STOMACH The sling fibres of the stomach hook around the notch between the lower end of the oesophagus on its left lateral aspect and the fundus of the stomach internal to the inner transverse layer (Figs 3 and 4) and then pass downwards, anteriorly and posteriorly, parallel to the lesser curve of the stomach. As stressed by Botha (1962), they are a reliable guide to the oesophago-gastric junction. This relationship between the sling fibres of the stomach and the oesophago-gastric junction is of considerable practical importance because these fibres may be recognized in living persons as a notch during a barium meal examination. In adult patients an abnormal location of this notch enables a confident diagnosis of sliding hiatal hernia to be made. This observation will be discussed in greater detail in a further paper.

THE CONSTRICTOR CARDIAE The constrictor cardiae is a small muscle band about the same size as a transverse muscle bundle, lying internal to the inner muscle layer, which completely encircles the lower end of the oesophagus. It runs from the left lateral aspect of the oesophagus above the upper limit of the sling fibres obliquely downwards and to the right, crossing over the transverse muscle bundles, and, about 1-2 cm. lower, changing its direction again and running parallel to the transverse muscle fibres of the lesser curve of the stomach (Fig. 4). This band was always found when searched for.

THE BRACKET FIBRES Internal to the transverse muscle layer the thin, branching, oblique bundles of the bracket fibres (Laimer's fibres) were found in about one-sixth of cases (Fig. 1a). 


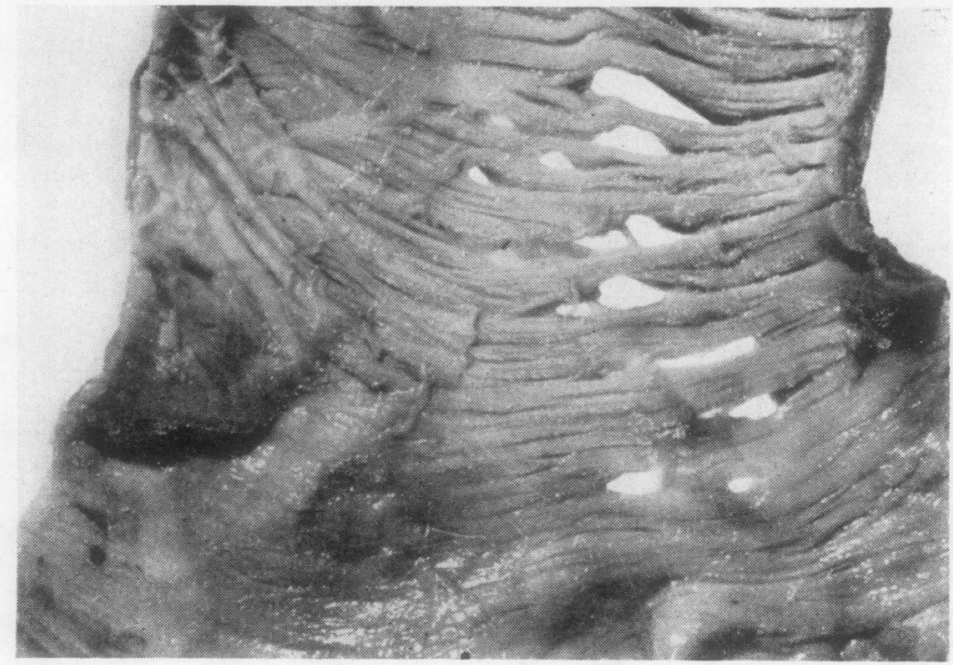

(a)

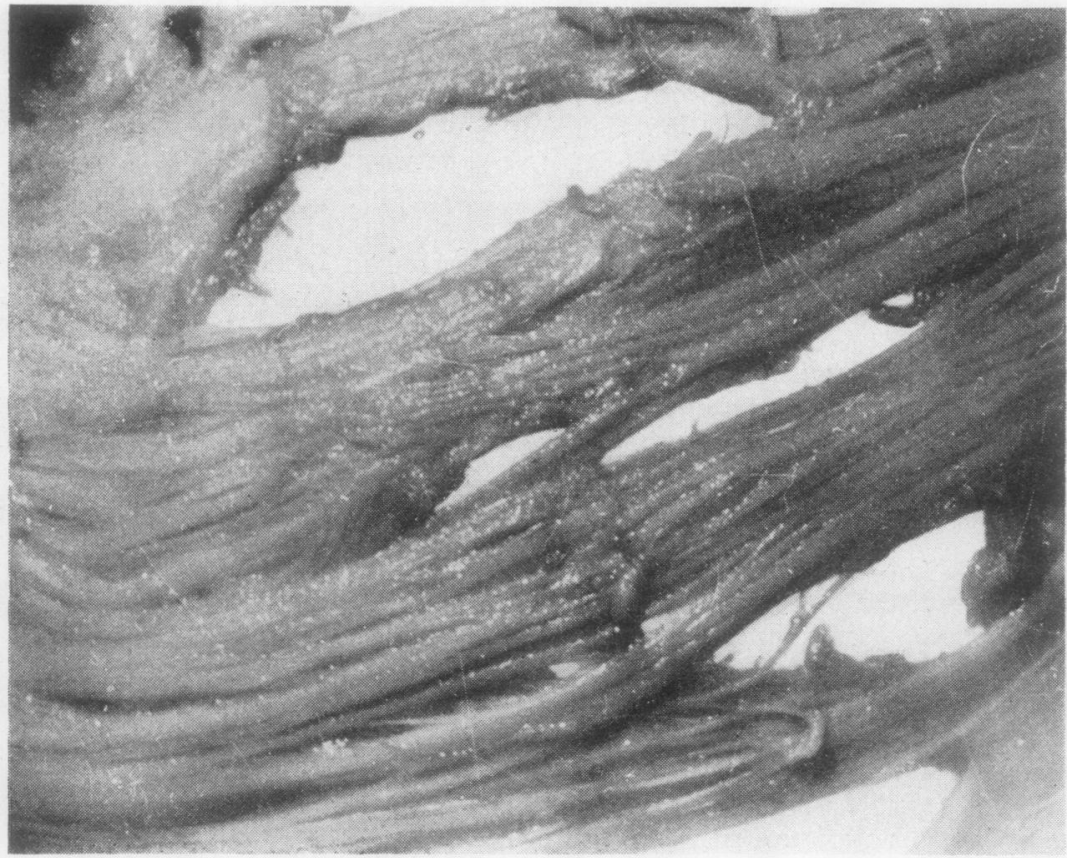

(b)

FIG. 1. The transverse muscle layer of the oesophagus and the bracket fibres. (a) Specimen of lower oesophagus opened longitudinally. The mucosa, the connective tissue between some of the transverse muscle bundles, and part of the longitudinal muscle layer have been removed to demonstrate that the transverse muscle layer is a reticular layer. Bracket fibres are visible in the upper part of the specimen on the viewer's left. (b) Close-up view of the same specimen showing the reticular arrangement of the muscle bundles. 


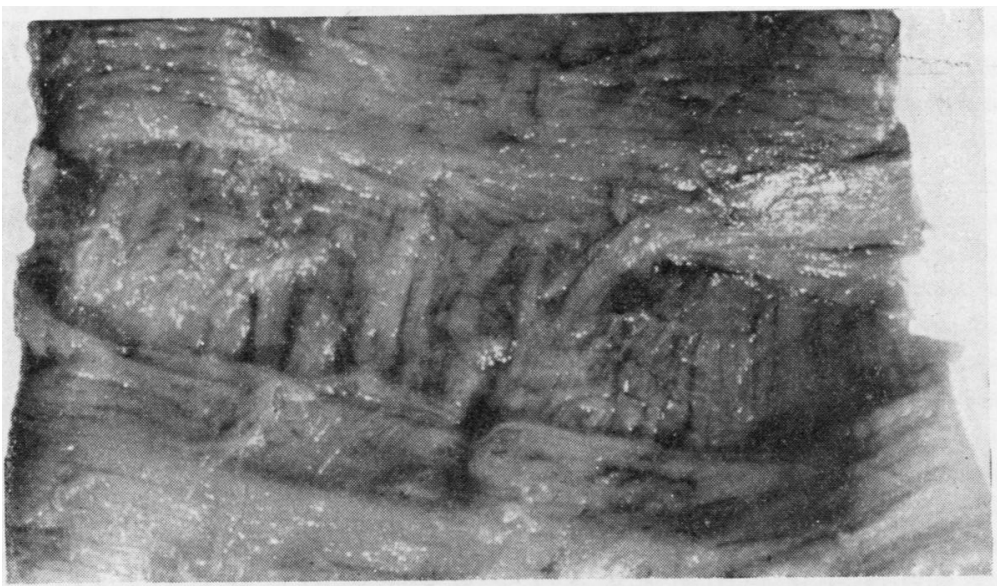

FIG. 2. The lower oesophagus has been everted and the mucosa and part of the transverse muscle layer removed. A muscle bundle of the transverse muscle layer on the viewer's right joins the longitudinal muscle layer.

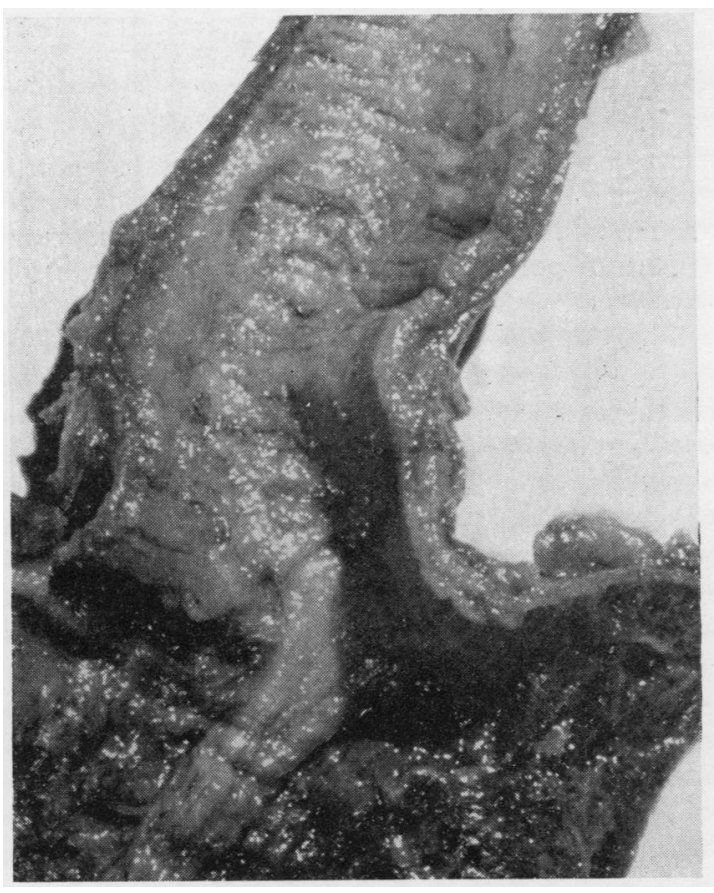

FIG. 3. Coronal section of the lower oesophagus and upper stomach showing the posterior parts of these structures. The mucosa has been removed. The sling fibres of the stomach lie in the notch between the lower end of the oesophagus and the fundus of the stomach. A partially contracted inferior oesophageal sphincter is present in the distal oesophagus.

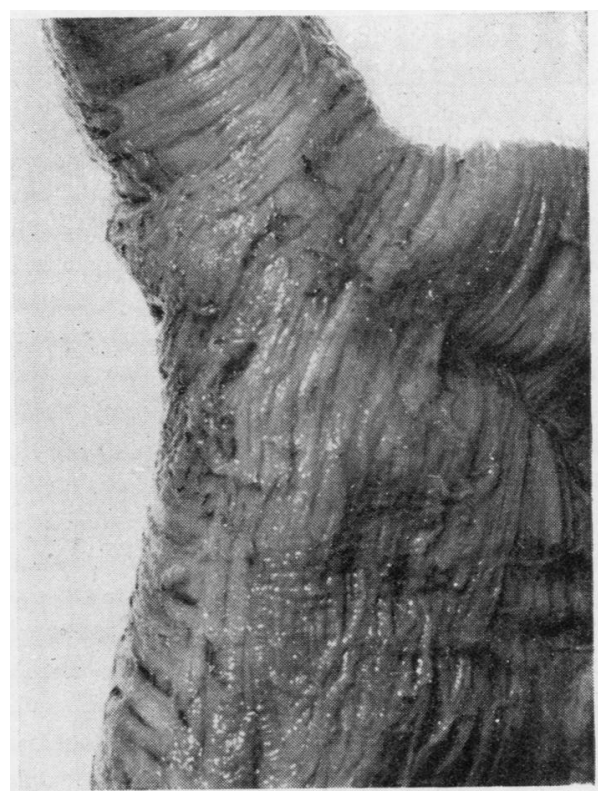

FIG. 4. The lower oesophagus and upper stomach have been everted and the mucosa removed. The lesser curve of the stomach lies on the viewer's left. Transverse muscle bundles are present in the lower oesophagus and the lesser curve of the stomach. The anterior band of the sling fibres runs internal to the transverse muscle layer parallel to the lesser curve of the stomach. Above the sling fibres and internal to the transverse muscle layer is the constrictor cardiae, which runs obliquely downwards across the transverse muscle layer towards the lesser curve of the stomach. 
An area of muscular contraction is recognized by thickening of the muscularis propria and the muscularis mucosae, large longitudinal mucosal folds, and narrowing or obliteration of the lumen.

According to these criteria, no evidence of muscular contraction in the oesophagus could be detected in just over one-half of the specimens, but two distinctly different parts of the oesophagus could be distinguished-namely, a long, proximal, tubular and a short, distal, fusiform portion. Radiographs of these specimens confirmed that the proximal tubular oesophagus expanded in a fusiform manner at its lower end (Fig. 5a).

In about one-third of the cases in this series a losalized area of contraction, 1-2 cm. above the hiatus, was found at the junction of a proximal tubular and distal fusiform part of the oesophagus. This is the 'inferior oesophageal sphincter' of Lerche (1950). When viewed from the stomach the inferior oesophageal sphincter may be seen as a distinct narrowing of the lumen (Fig. 6). On opening such a specimen by means of a longitudinal incision, one may be surprised to discover that a considerab!e narrowing of the lumen was caused by a now insignificant-looking ridge (Figs 3 and 7). There are two important reasons for this apparent paradox: (1) For every $10 \%$ increase or decrease in a circumference of a circle, the area of the circle increases or decreases by

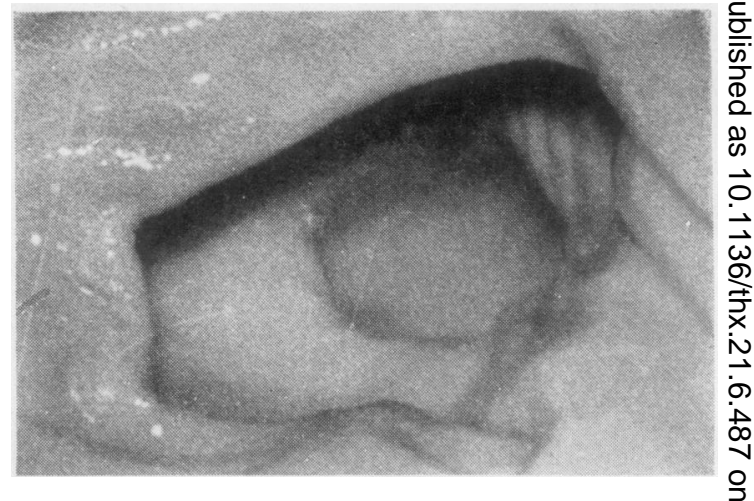

FIG. 6. Same specimen as in Fig. 5b. Lower oesophagus viewed from the stomach. The inferior oesophageal $Z$ sphincter causes a narrowing of the lumen above the relaxed vestibule.

$21 \%$ respectively. (2) A relatively small muscular $\overrightarrow{\mathscr{C}}$ contraction causes the oesophageal mucosa to form large, longitudinal fo.ds, and it is these folds which eventually narrow or obliterate the lumen. Hence, areas of contraction are best judged by means of transverse sections (Figs $8 \mathrm{a}, \mathrm{b}$, and c). Radiographs of these barium-distended specimens revealed that the inferior oesophageal sphincter causes a cons.riction in the form of a ring with a rounded margin at the junction of the tubular and fusiform portions of the oesophagus (Fig. 5b).

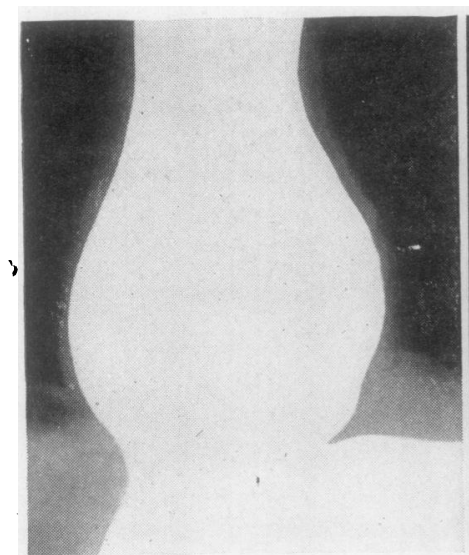

(a)

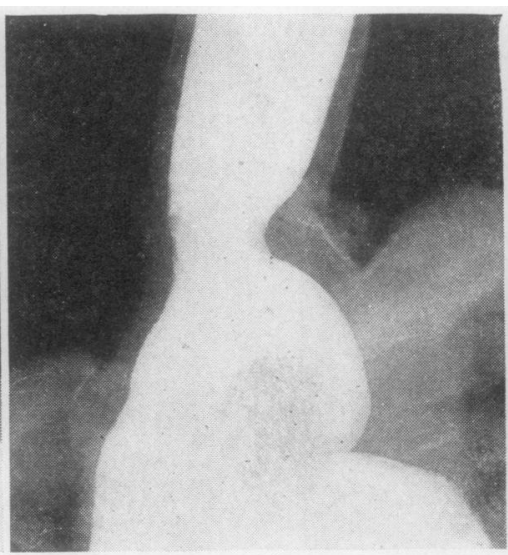

(b)

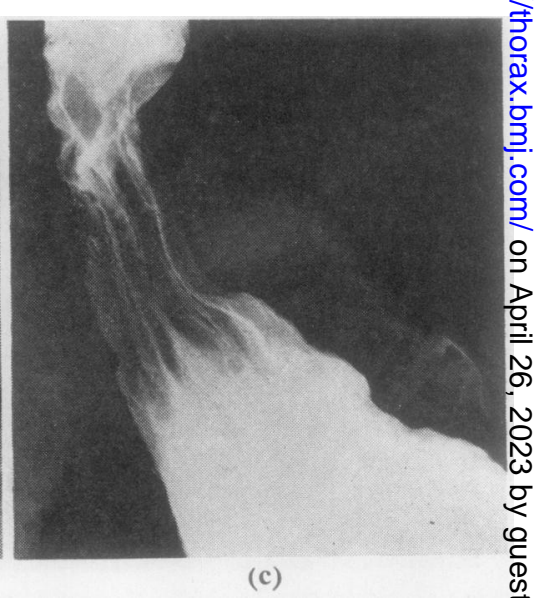

FIG. 5. Specimens of oesophagus and stomach distended with barium. (a) Inferior oesophageal sphincter and vestibule relaxed. The proximal tubular oesophagus expands in a fusiform manner at its lower end. The transverse mucosal fold between the lower end of the oesophagus and the fundus of the stomach forms a $\overrightarrow{\mathbb{D}}$ ledge-like filling defect. (b) Inferior oesophageal sphincter partially contracted and vestibule relaxed. The sphincter causes a contriction in the form of a ring with a rounded margin. Note the filling defect caused by $\mathbb{\circledR}^{\circ}$ the transverse mucosal fold. (c) Inferior oesophageal sphincter and vestibule contracted. Barium caught in the grooves between the longitudinal folds in the contracted vestibule forms longitudinal linear shadows. 

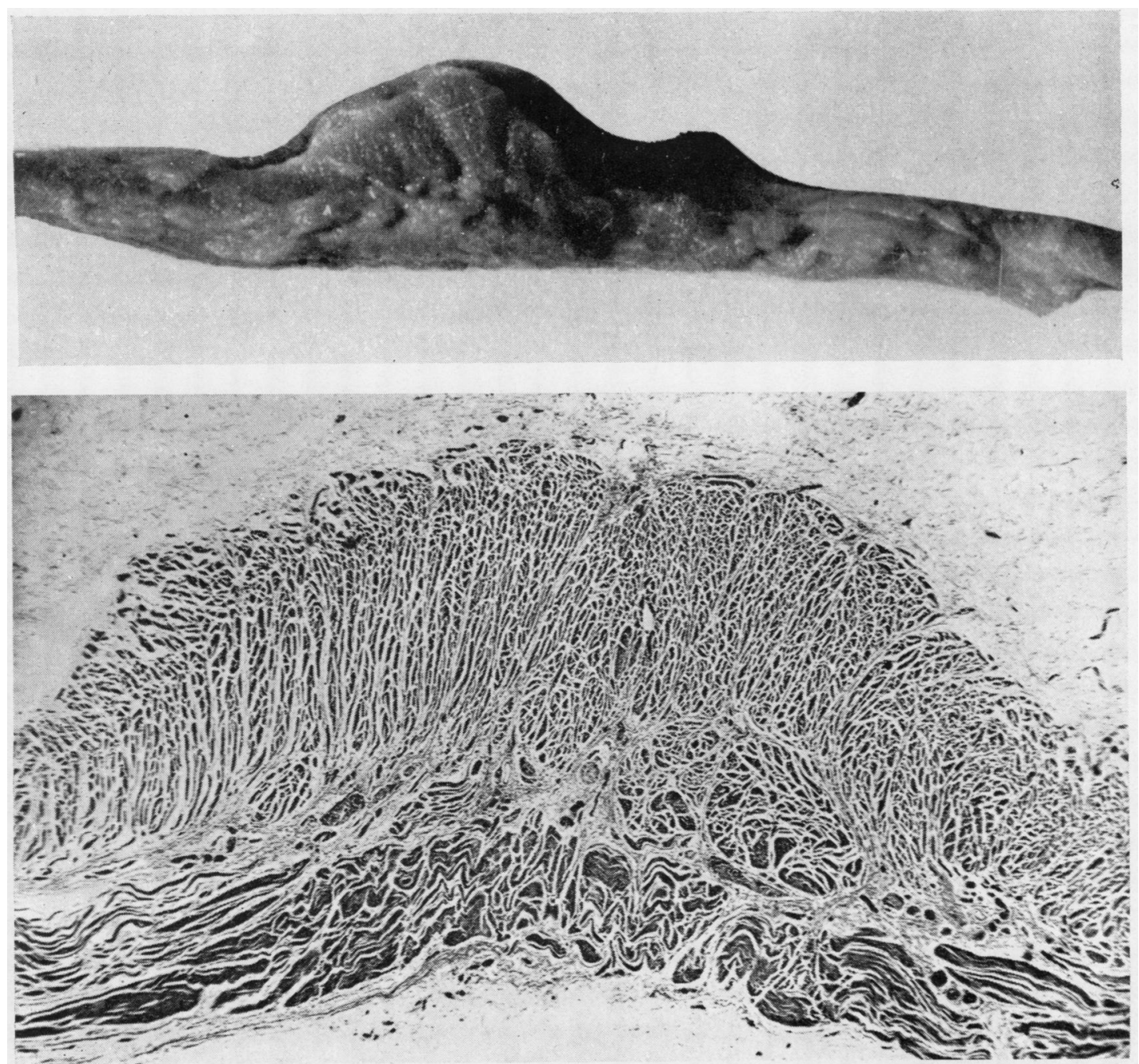

FIG. 7. (Above) Longitudinal section through inferior oesophageal sphincter. (Below) Histological section of same specimen showing thickening of the muscularis propria.

The term 'vestibule' is used to denote the potentially more distensible portion of the terminal oesophagus between the inferior oesophageal sphincter and the sling fibres of the stomach, or between the junction of the tubular and fusiform distal oesophagus and the sling fibres of the stomach.

Both the inferior oesophageal sphincter and the vestibule were found to be contracted in approximately one-sixth of the cases, i.e., the transverse and longitudinal muscle layer and the muscularis mucosae were thickened between the inferior oesophageal sphincter and the sling fibres of the stomach, and prominent mucosal folds occluded the lumen. Contraction of the inferior oesophageal sphincter and the vestibule was recognized on radiographs of the specimens by a long narrow segment at the distal end of the oesophagus in which the barium caught in the grooves between the longitudinal mucosal folds was seen as longitudinal linear shadows, usually three in number (Fig. 5c).

Berridge (1961) has described three similar radiographic appearances seen during cinefluorographic studies of the distal oesophagus in adult patients. The sequence of events which he observed was as follows: (1) The tubular oesophagus was seen to become continuous with a 


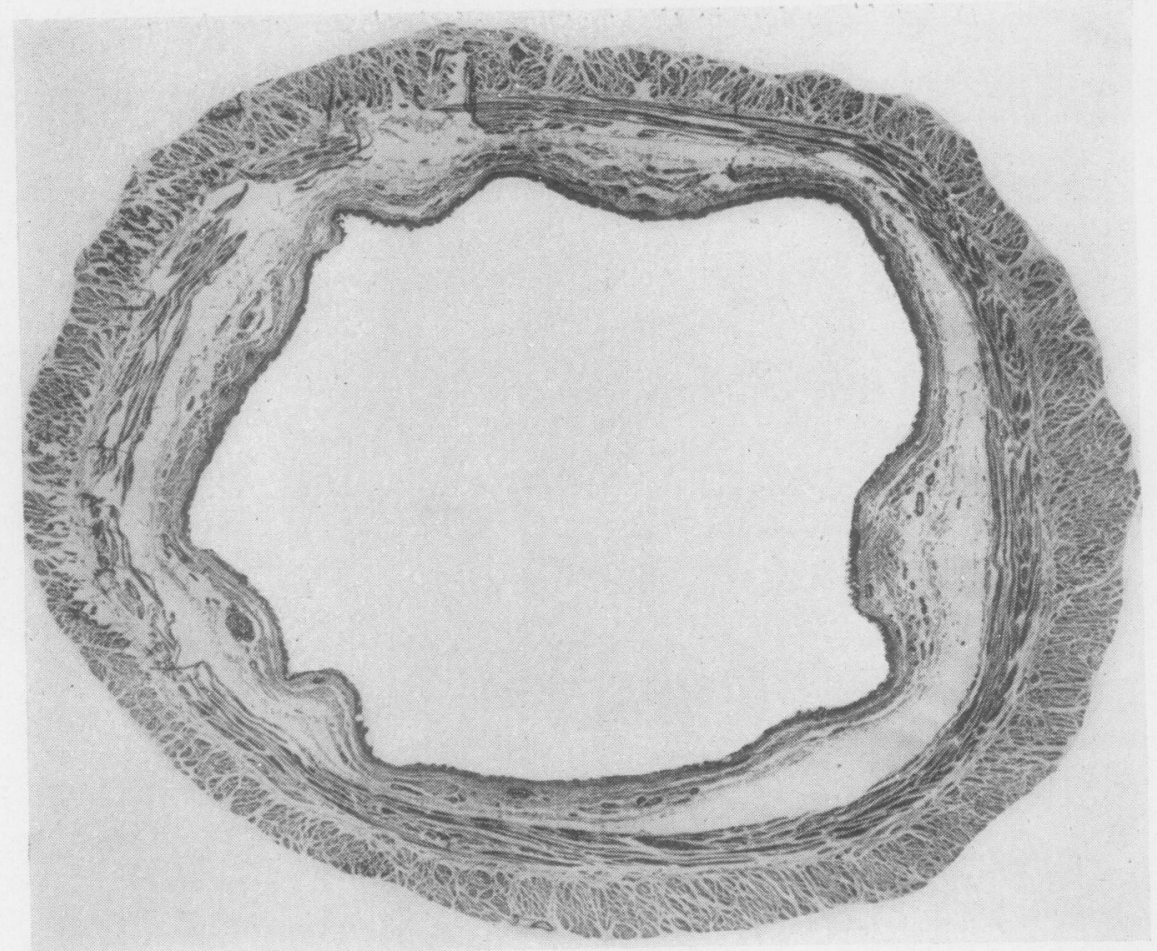

(a)

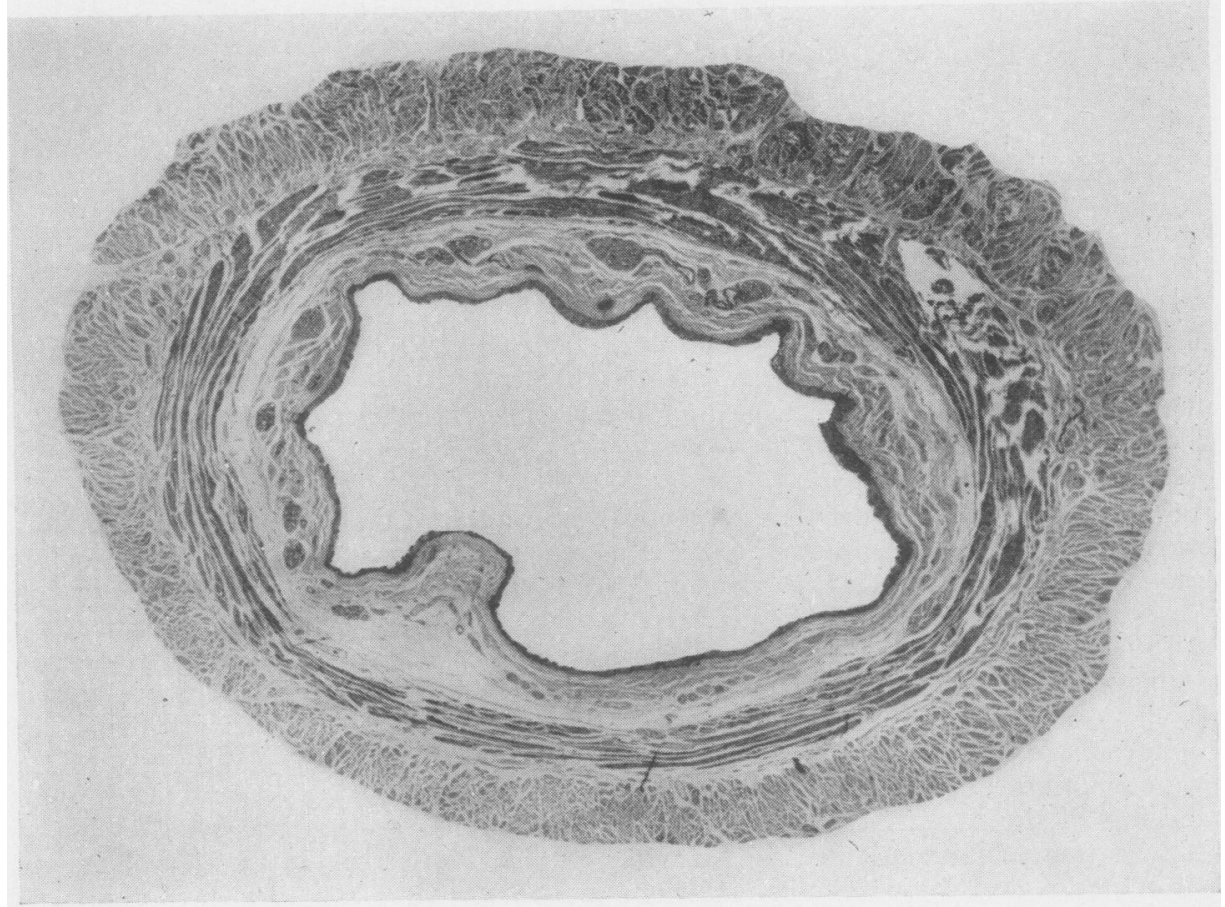

(b)

FIG. 8 


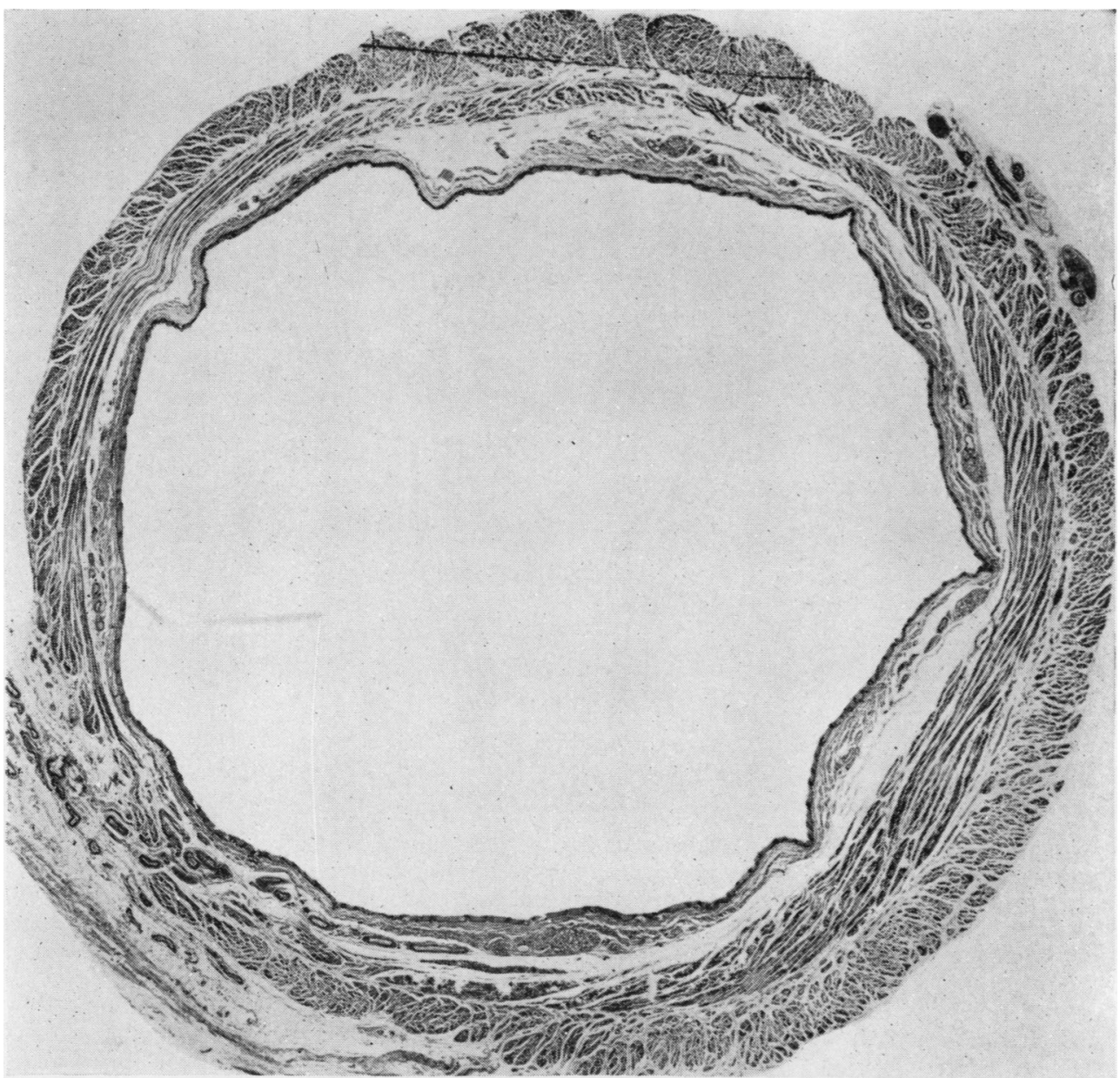

(c)

FIG. 8. Same specimen as in Fig. 5b. Transverse histological sections (a) above inferior oesophageal sphincter, (b) at inferior oesophageal sphincter; thickening of both layers of the muscularis propria and the muscularis mucosae, some longitudinal folding of the mucosa, and narrowing of the lumen; (c) below inferior oesophageal sphincter. The inferior oesophageal sphincter in this specimen is only moderately contracted.

dilated fusiform distal end. No constriction was seen between the tubular and fusiform portions. (2) A ring appeared between the tubular and fusiform segments. (3) The fusiform segment contracted, becoming narrow and tubular with prominent longitudinal mucosal folds.

In the past, three conflicting views have been expressed concerning sphincters in the distal oesophagus, namely (1) that no sphincter exists, (2) that there is a short sphincter situated $1-2 \mathrm{~cm}$. above the hiatus (the inferior oesophageal sphincter), and (3) that there is a long sphincter extending for several centimetres from the oesophago- gastric junction proximally. In fact, all three appearances exist and are merely different stages of the complex action that occurs in the distal oesophagus during swallowing. The first appearance is due to relaxation of the sphincter and the vestibule, the second to contraction of the inferior oesophageal sphincter above a relaxed vestibule, and the third to contractions of both the sphincter and the vestibule (Fig. 9). The inferior oesophageal sphincter is a physiological sphincter, for there is good anatomical and radiological evidence that it is capable of contracting independently from the rest of the oesophagus, and, when 


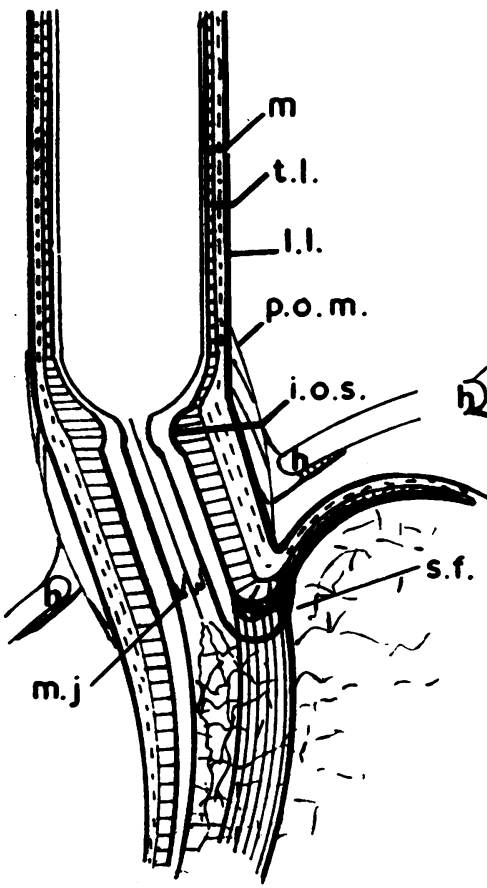

1

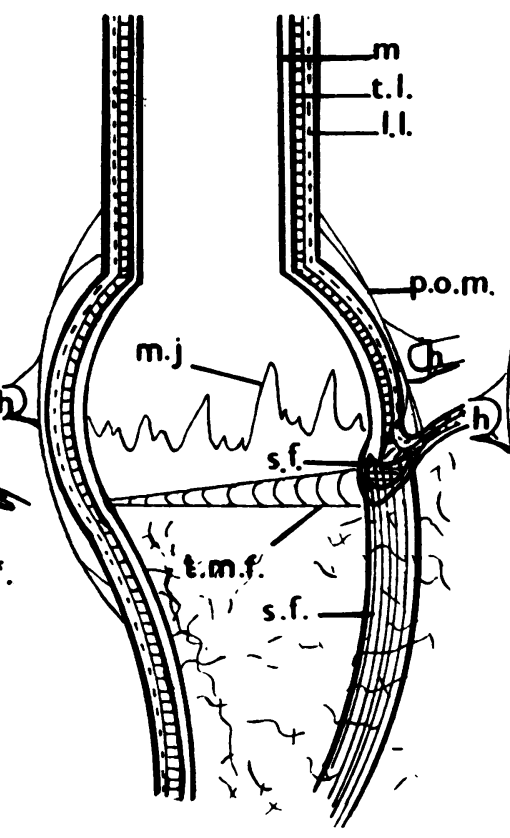

2

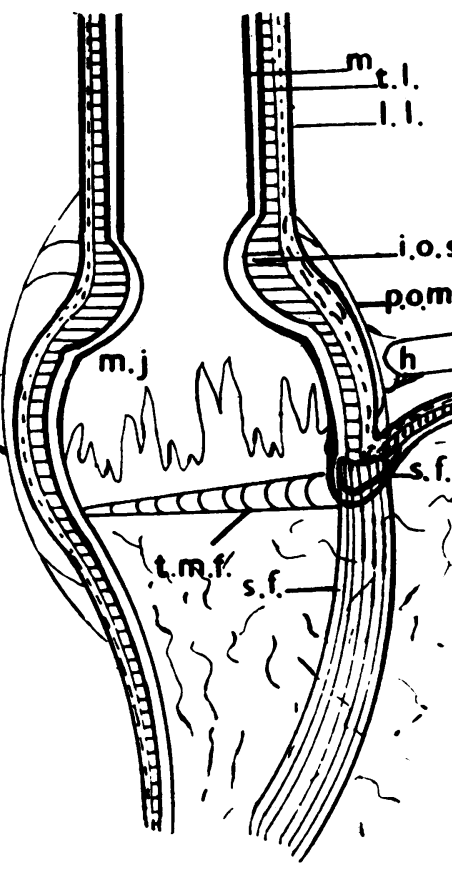

3

FIG. 9. Diagrammatic representation of the anatomy of the lower oesophagus and upper stomach showing $\stackrel{\mathbb{Q}}{\Rightarrow}$ (1) vestibule contracted, (2) vestibule relaxed, (3) inferior oesophageal sphincter contracted, vestibule relaxed $\overrightarrow{\overrightarrow{0}}$ m, mucosa; m.j., mucosal junction; t.m.f., transverse mucosal fold ; t.l., transverse muscle layer ; l.l., longi ${ }^{3}$

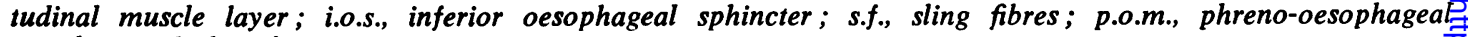
membrane; h, hiatal margin.

relaxed, no trace of it can be found. Similarly, the vestibule when contracted forms a sphincter. Radiological studies in the living indicate that these two sphincters, possibly reinforced by a third, the sling fibres, act together in regulating flow through the distal oesophagus into the stomach and in closing it. This will be discussed in a further paper.

\section{THE MUCOSA OF THE OESOPHAGUS}

THE MUCOSAL JUNCTION The junction between the squamous epithelium of the oesophagus and the columnar epithelium is clearly visible to the naked eye as an irregular line, the well-known Z-line (Fig. 10). This mucosal junction is variable in position and length. So great is this variation that the mucosal junction is no guide to the position of the oesophago-gastric junction.

A mixture of gelatin, barium, and India ink was injected into the submucosa of the oesophagus and allowed to gravitate downwards. In 19 cases where it had been injected in the correct plane the mixture was arrested at the mucosal junction: This finding indicates that the mucosa is firmly. attached to the muscle layers at the mucosab junction.

LONGITUDINAL MUCOSAL FOLDS Both in the regions lined by squamous and by columnar epitheliumo the mucosal folds of the oesophagus are longitu dinal in areas of muscular contraction and when the oesophagus is relaxed and not distended 0 (Fig. 10). On a radiograph of a specimen, oeso N phageal squamous and columnar epithelium areog indistinguishable.

The mucosa is attached more firmly to the underlying muscle layers in the grooves between? the longitudinal mucosal folds. When the mucosato is stripped off the muscle layer, greater resistanceo to stripping is encountered along the longitudinat

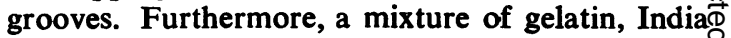
ink, and barium injected into the submucosa tends ${ }^{\circ}$ to run longitudinally for several centimetres along 


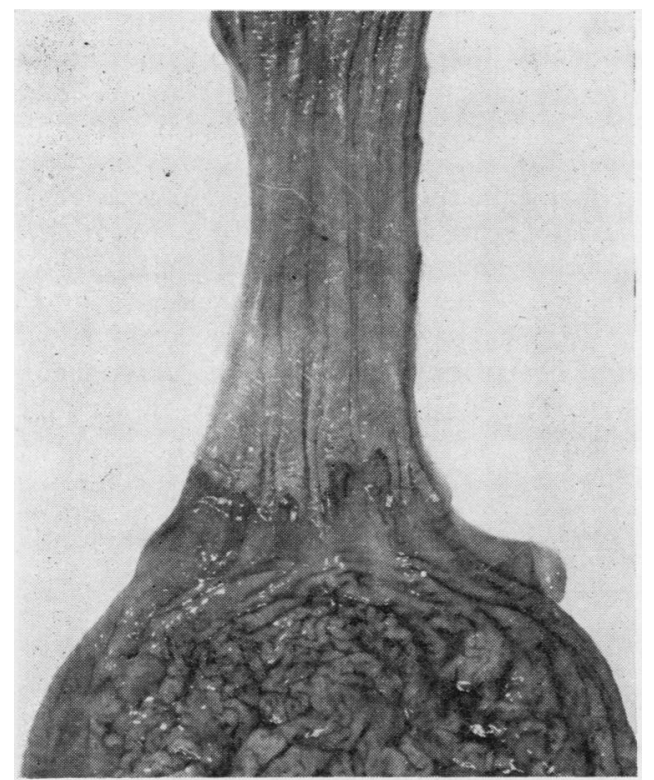

FIG. 10. Fresh specimen of lower oesophagus and upper stomach opened longitudinally. The mucosal junction lies well above the oesophago-gastric junction. The longest digitation of squamous epithelium measured $1.5 \mathrm{~cm}$. Longitudinal mucosal folds of the oesophagus are present both in the regions lined by squamous and by columnar epithelium. Gastric columnar epithelium forms rugae.

a fold between two grooves before spreading laterally. This attachment of the mucosa to the muscle along the longitudinal grooves is not due to the deep oesophageal glands and ducts, as oft quoted in the literature, for histological examination reveals that the glands and ducts may lie both in the grooves and in the folds.

Serial sections of distended gullets show that the mucosa is flattened in areas where the muscles are relaxed and thrown into longitudinal folds in areas of muscular contraction. The attachment of the mucosa to the muscle layer at fixed points along the circumference of the wall of the oesophagus encourages the formation of longitudinal folds as the muscularis propria contracts. A relatively small change in the lumen of the muscle layer results in a large change in the lumen of the oesophagus, because the longitudinal folds rapidly increase in size as the muscular ring narrows. These folds produce a stellate oesophageal lumen on cross-section. A further decrease in the diameter of the muscular ring now obliterates this stellate lumen by increasing the size of the folds and also by pressing the folds together. At this stage, a surprisingly substantial proportion of the wall of the oesophagus is formed by the mucosa. Remove this mucous membrane plug, and a gaping hole remains. The mucosa and muscle thus act in unison in closing the lumen, the speed of closure and mechanical effectiveness of muscular contraction being enhanced by the mucosa.

As the diameter of the distended, relaxed vestibule exceeds that of the rest of the oesophagus, it has a greater area of mucosa per unit length, so particularly fleshy folds form when it contracts.

TRANSVERSE MUCOSAL FOLDS When specimens in which the vestibule is relaxed are distended, an almost horizontal mucosal fold forms at the level of the sling fibres of the stomach (Fig. 11). This fold lies below the mucosal junction in the majority of cases. In barium-filled specimens, the transverse fold forms a ledge-like ring at the oesophago-gastric junction, usually most prominent and sometimes only on the left (Figs 5a and b). Radiological studies in living persons have shown that a similar ring may form at the same level as the notch caused by the sling fibres of the

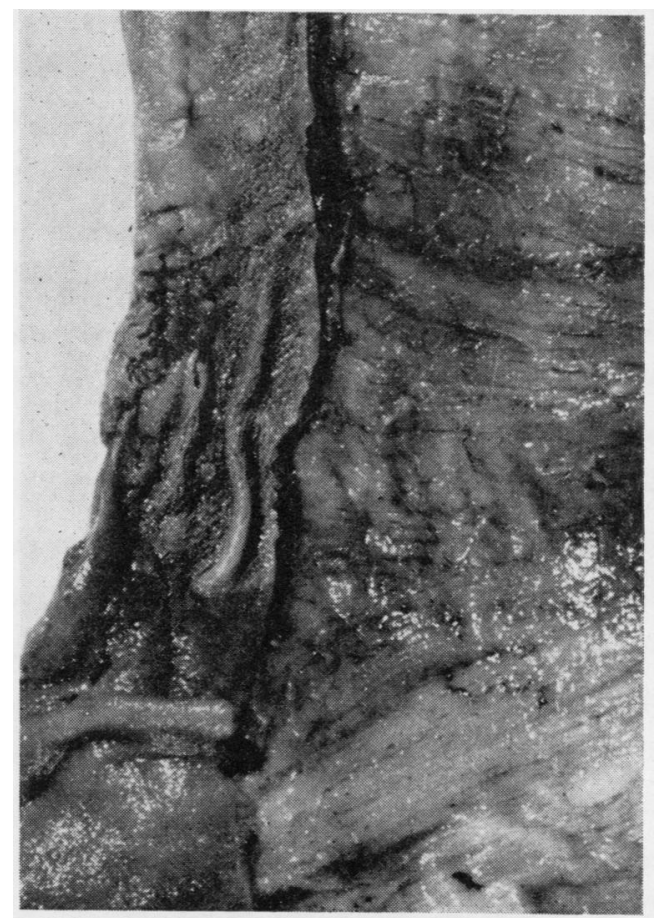

FIG. 11. Specimen of lower oesophagus and upper stomach opened longitudinally. Part of the mucosa has been removed demonstrating the relation between the sling fibres of the stomach, the transverse mucosal fold, and the mucosal junction. 
stomach in patients with a hiatal hernia. This subject will be discussed more fully in a further paper.

Frequent reference is made in the literature to a 'mucosal junction ring'. All illustrations show this ring to have a perfectly straight outline. It has long been accepted that the mucosal junction is not a straight line but has digitations of varying length. If this ring were to follow the line of the mucosal junction it, too, should have a jagged outline on radiographs. This is not the case.

The transverse mucosal fold lies at the oesophago-gastric junction and not in the oesophagus. When oesophageal squamous epithelium is gripped with a pair of forceps and raised, it never forms a transverse mucosal fold in a fresh specimen irrespective of the mode of traction. A flat, triangular fold always forms with its long base running parallel to the longitudinal folds of the oesophagus and its apex at the point at which the traction is being applied. Gastric mucosa, on the other hand, either assumes a stellate configuration, or the base on the triangle may be dependent on the direction of the gripping force.

Histological sections of the transverse mucosal fold show that in most cases it is covered by columnar epithelium. Rarely, a digitation of the squamous epithelium may run on to the base of the fold and a histological section taken at this point shows part or all of the upper surface of the fold to be covered by squamous epithelium and the rest by columnar epithelium. In only one case did a digitation of squamous epithelium cover the whole of the fold. The site at which the section is taken thus determines the histological appearance. Similarly, in living persons, the type of epithelium found on histological examination will depend on the exact point in the circumference of the oesophagus at the particular level at which the biopsy is taken; this is particularly true in persons who have a Z-line with long digitations.

\section{ANATOMICAL STRUCTURES WHICH KEEP THE STOMACH IN THE ABDOMEN}

The oesophagus is attached to the diaphragm by a fibro-elastic membrane known as the phrenooesophageal membrane or ligament. The normal oesophagus in an adult patient can slide up and down in the hiatus over a length of about $2 \mathrm{~cm}$. When the nerves, vessels, peritoneum, and fat attached to the stomach are cut it is not possible, by pulling on the oesophagus, to displace the sling fibres of the stomach above the hiatus. However, once the attachments of the phreno-oesophageal membrane to the oesophagus are cut, the sling 음 fibres of the stomach can be pulled into the chest.

After arising from the under surface of the $\stackrel{\mathbb{Q}}{\square}$ diaphragm the phreno-oesophageal membrane o divides into two limbs. The upper limb passes through the hiatus, where it is reinforced by the supradiaphragmatic fascia before inserting by $\vec{\omega}$

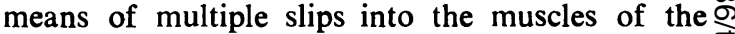
oesophagus. The lower limb is inserted into the $\overrightarrow{\vec{x}}$ lower oesophagus and upper stomach below the $N$ hiatus. The membrane allows the oesophagus to slide up and down in the hiatus rather like a tendon in a tendon sheath.

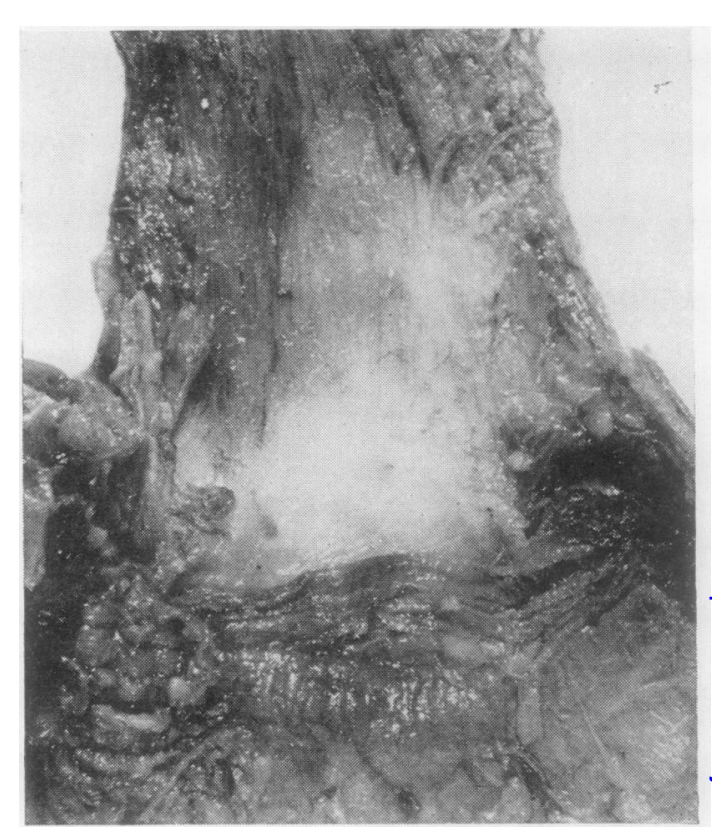

FIG. 12. Specimen of diaphragm, phreno-oesophageai membrane, and lower oesophagus. The structures visible을 are the upper surface of the diaphragm immediately $D$ adjacent to the hiatus in the lower part of the photograph, the upper limb of the phreno-oesophageal membrane as a whitish area, and the external surface of the oesophagus. The line of insertion of the upper limb of the phreno-N oesophageal membrane is very irregular.

The line of insertion is irregular. Some digitations of the membrane may extend for up to $2 \mathrm{~cm}$. further along the wall than adjacent digitations. ${ }^{-}$ (Fig. 12). Contrary to some current views, there is no anatomical evidence that the inferior oeso- $\frac{}{\mathbb{D}}$ phageal sphincter is not a sphincter at all but is caused by traction on the wall of the oesophagus $\triangle$ by the upper limb of the phreno-oesophagealo membrane, for the digitations of the membraneo 
are inserted below, into, or above the sphincter. Furthermore, the behaviour of the sphincter in living persons indicates that it is due to a localized contraction of the muscle layers. Similarly, the ring caused by the transverse mucosal fold is not due to traction of the lower limb of the phrenooesophageal membrane. As a corollary, the upper and lower limbs of the phreno-oesophageal membrane do not define the limits of the vestibule.

\section{HIATAL HERNIA}

Seven cases of hiatal hernia were encountered in the series, one of which had an oesophagus almost entirely lined by columnar epithelium and several penetrating ulcers on the postero-lateral wall of the oesophagus. Only the case of columnar-cell lining of the oesophagus was diagnosed during life.

Common to all the hernia cases was the presence of $a$ thin and lengthened phreno-oesophageal membrane. Indeed, in some the phreno-oesophageal membrane could not be identified. In all, the sling fibres of the stomach could be drawn well above the hiatus by pulling on the oesophagus.

On superficial inspection, the oesophagus in the clinically unsuspected cases did not appear grossly abnormal, but on close inspection small superficial mucosal scars could be detected in some. Difficulty was experienced in stripping the mucosa off the muscle layers, and on histological examination submucous fibrosis was present in all. It is likely that these unsuspected cases would have been overlooked during routine post-mortem examination. All specimens with a hiatal hernia (excluding the case of columnar-cell lining of the oesophagus) had a relaxed vestibule above the sling fibres and the transverse mucosal fold, and in none was the inferior oesophageal sphincter contracted.

The incidence of hiatal hernia in this series was lower than is commonly reported by radiologists, possibly because minimal hiatal herniation shown radiologically is not associated with appreciable weakening of the phreno-oesophageal membrane. Unusual prominence of the sling fibres of the stomach was observed in the cases of unsuspected hiatal hernia but not in the case with an oesophagus lined by columnar epithelium. As the sling fibres of the stomach are easily identified at necropsy, a simple post-mortem test for hiatal hernia is now available. If the phreno-oesophageal membrane is left undisturbed and the sling fibres of the stomach can be pulled above the hiatus, a hiatal hernia is present.

\section{SUMMARY}

Some anatomical findings in 132 post-mortem specimens of oesophagus, stomach, and diaphragm are discussed. There were seven cases of hiatal hernia, one of which had most of the oesophagus lined by columnar epithelium.

The transverse muscle layer of the muscularis propria is a reticular layer and contributes muscle bundles to the longitudinal layer. Both muscle layers contract together, simultaneously shortening and narrowing the contracted segment and causing the mucosa to form large longitudinal folds. In the notch between the left lateral aspect of the lower end of the oesophagus and the fundus of the stomach lie the sling fibres of the stomach. As they are a reliable guide to the oesophagogastric junction, displacement of these fibres above the hiatus enables a diagnosis of hiatal hernia to be made post mortem and on barium studies in living persons.

In just over half the cases there was no evidence of muscular contraction in the oesophagus, and the tubular oesophagus expanded in a fusiform manner at its lower end before entering the stomach at the level of the sling fibres. A localized area of contraction at the upper limit of the vestibule (the inferior oesophageal sphincter) was present in about one-third. The inferior oesophageal sphincter and the whole vestibule were contracted in the remaining cases. It is thus clear why there has been so much controversy in the literature over the sphincters in the lower oesophagus. Each of these appearances is merely a phase of the normal action seen in the distal end of the oesophagus during swallowing (Fig. 9).

The variability of the mucosal junction has been confirmed. Longitudinal folds form in the oesophagus when the muscles contract, bicause the mucosa is attached to the muscle wall at fixed points in its circumference. These folds play an important part in the closure of the oesophageal lumen. A transverse mucosal fold forms at the same level as the sling fibres of the stomach when the vestibule is relaxed and distended. This fold is not related to the mucosal junction.

The oesophagus is anchored to the hiatus by the phreno-oesophageal membrane, which permits the oesophagus to slide up and down in the hiatus and prevents the stomach from herniating into the chest. Like the mucosal junction, this membrane has irregular digitations and thus does not define the limits of the vestibule or form either the inferior oesophageal sphincter or the mucosal fold at the level of the sling finres of the stomach. In 
cases of sliding hiatal hernia the phreno-oesophageal membrane is attenuated.

The way in which various structures at the distal end of the oesophagus behave in living persons will be fully discussed in a further paper.

We wish to thank the British Medical Research Council for their generous grant to Dr. G. W. Friedland, who carried out the macroscopic anatomical dissections, Dr. Ifor Williams for suggesting some of the experiments on the mucosa, and the Department of Photography, Addenbrooke's Hospital, for the photographs.

\section{REFERENCES}

Berridge, F. R. (1961). The mechanism at the cardia: A symposium. $\vec{\circ}$ III. Radiological aspects. Brit. J. Radiol., 34, 487. Botha, G. S. Muller (1962). The Gastro-oesophageal Junction, p. 5. $\overrightarrow{\vec{\omega}}$
Churchill, London.

Lerche, W. (1950). The Esophagus and Pharynx in Action, p. 55. Thomas, Springfield, Illinois. 